\title{
Polarization measurement of free electron laser pulses in the VUV generated by the variable polarization source FERMI
}

\author{
P. Finetti, ${ }^{* 1 a}$ E. Allaria, ${ }^{a}$ B. Diviacco, ${ }^{a}$ C. Callegari, ${ }^{a}$ B. Mahieu,,${ }^{b}$ J. Viefhaus,${ }^{c}$ M. Zangrando,${ }^{a, i}$ G. \\ De Ninno, ${ }^{\mathrm{a}, \mathrm{d}}$ G. Lambert, ${ }^{\mathrm{b}}$ E. Ferrari, ${ }^{\mathrm{a}, \mathrm{e}} \mathrm{J}$. Buck, ${ }^{\mathrm{k}}$ M. Ilchen,,${ }^{\mathrm{k}, \mathrm{m}}$ B. Vodungbo,,${ }^{\mathrm{g}, \mathrm{h}}$ N. Mahne,${ }^{\mathrm{a}} \mathrm{C}$. \\ Svetina,,${ }^{\mathrm{a}, \mathrm{e}}$ C. Spezzani, ${ }^{\mathrm{a}}$ S. Di Mitri, ${ }^{\mathrm{a}}$ G. Penco, ${ }^{\mathrm{a}}$ M. Trovò, ${ }^{\mathrm{a}}$ W.M. Fawley, ${ }^{\mathrm{a}}$ P. Rebernik,,${ }^{\mathrm{a}, \mathrm{d}}$ D. \\ Gauthier, ${ }^{\mathrm{a}, \mathrm{d}}$ C. Grazioli, ${ }^{\mathrm{d}, \mathrm{a}, \mathrm{i}}$ M. Coreno, ${ }^{\mathrm{a}, \mathrm{j}}$ B. Ressel, ${ }^{\mathrm{d}}$ A. Kivimäki, ${ }^{\mathrm{i}}$ T. Mazza, ${ }^{\mathrm{k}}$ L. Glaser, ${ }^{\mathrm{c}}$ F. Scholz, ${ }^{\mathrm{c}}$ \\ J. Seltmann, ${ }^{\mathrm{c}}$ P. Gessler, ${ }^{\mathrm{k}}$ J. Grünert, ${ }^{\mathrm{k}}$ A. De Fanis, ${ }^{\mathrm{k}}$ M. Meyer, ${ }^{\mathrm{k}}$ A. Knie, ${ }^{1}$ S. P. Moeller, ${ }^{\mathrm{f}}$ L. \\ Raimondi, ${ }^{a}$ F. Capotondi, ${ }^{a}$ E. Pedersoli, ${ }^{a}$ O. Plekan, ${ }^{a}$ M. Danailov, ${ }^{a}$ A. Demidovich, ${ }^{a}$ I. Nikolov, ${ }^{a}$ A. \\ Abrami, ${ }^{\mathrm{a}}$ J. Gautier, ${ }^{\mathrm{b}}$ J. Lüning,,${ }^{\mathrm{g}, \mathrm{h}}$ P. Zeitoun, ${ }^{\mathrm{b}}$ and L. Giannessi ${ }^{\mathrm{a}, \mathrm{n}}$
}

aElettra-Sincrotrone Trieste, Basovizza I-34149, Italy; 'baboratoire d'Optique Appliquée, ENSTA ParisTech - CNRS UMR 7639 - Ecole polytechnique, Chemin de la Hunière, 91761 Palaiseau, France; ${ }^{\mathrm{c} D E S Y, ~ F S-P E, ~ N o t k e s t r a s s e ~ 85, ~} 22607$ Hamburg, Germany; ${ }^{\mathrm{d}}$ Laboratory of Quantum Optics, University of Nova Gorica, Ajdovščina 5270 - Slovenia; 'University of Trieste, Graduate School of Nanotechnology, Piazzale Europa 1, 34127 Trieste, Italy; fSLAC National Accelerator Laboratory, 2575 Sand Hill Road, Menlo Park, CA 94025, USA; 'Sorbonne Universités, UPMC Univ Paris 06, UMR 7614, LCPMR, 75005 Paris, France; ${ }^{\mathrm{h}} \mathrm{CNRS}$, UMR 7614, LCPMR, 75005 Paris, France; ${ }^{\mathrm{i}} \mathrm{CNR}$ Istituto Officina dei Materiali, Laboratorio TASC, 34149 Basovizza, Trieste, Italy; ${ }^{\mathrm{j} C N R}$-ISM, UOS RM1, CP10, I-00019 Monterotondo Scalo (RM), Italy; kEuropean XFEL, Albert-Einstein-Ring 19,

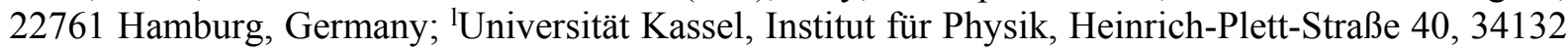
Kassel, Germany; ' Stanford PULSE Institute, 2575 Sand Hill Road, SLAC National Accelerator Laboratory, Menlo Park, California 94025, USA; "ENEA, Via Enrico Fermi, 45, 00044 Frascati Roma, Italy.

\begin{abstract}
FERMI, based at Elettra (Trieste, Italy) is the first free electron laser (FEL) facility operated for user experiments in seeded mode. Another unique property of FERMI, among other FEL sources, is to allow control of the polarization state of the radiation. Polarization dependence in the study of the interaction of coherent, high field, short-pulse ionizing radiation with matter, is a new frontier with potential in a wide range of research areas. The first measurement of the polarization-state of VUV light from a single-pass FEL was performed at FERMI FEL-1 operated in the $52 \mathrm{~nm}-26 \mathrm{~nm}$ range. Three different experimental techniques were used. The experiments were carried out at the end-station of two different beamlines to assess the impact of transport optics and provide polarization data for the end user. In this paper we summarize the results obtained from different setups. The results are consistent with each other and allow a general discussion about the viability of permanent diagnostics aimed at monitoring the polarization of FEL pulses.
\end{abstract}

Keywords: polarization, polarimetry, FEL, VUV

* paola.finetti@elettra.eu, phone +39040 3758598, http://www.elettra.trieste.it

X-Ray Free-Electron Lasers: Beam Diagnostics, Beamline Instrumentation, and Applications II, edited by Stefan P. Hau-Riege, Stefan P. Moeller, Makina Yabashi, Proc. of SPIE Vol. 9210,

92100K · (c) $2014 \mathrm{SPIE} \cdot \mathrm{CCC}$ code: 0277-786X/14/\$18 · doi: 10.1117/12.2062717

Proc. of SPIE Vol. $921092100 \mathrm{~K}-1$ 


\section{INTRODUCTION}

The last decade was characterized by a tremendous progress in the development and achievement of short wavelength free electron lasers and FERMI, based at Elettra, Trieste-Italy, is the first FEL facility operated for users experiments in seeded mode $[1,2]$. With this mode of operation, coherent emission is induced by the interaction of the electron bunch with the electromagnetic field of an external laser and then amplified by an array of undulators. FERMI can deliver high power ultra-short VUV and soft X-ray pulses, up to multi GW peak power over a pulse duration of the order of $100 \mathrm{fs}$, with a high level of wavelength and power stability [1,2]. The wavelength tuning of the seed laser (an optical parametric amplifier, OPA) combined with the use of variable gap undulators allows generation of FEL pulses spanning a continuous wavelength range. Seeding is beneficial also to the longitudinal and transverse coherence of the emitted light $[1,2]$.

Another prominent feature that distinguishes FERMI from other short-wavelength FEL is the possibility to control the polarization of the radiation. This type of control is achieved by using specially designed undulators that can change the trajectory of the electron beam, both in the vertical and in the horizontal direction. FERMI is designed around the AppleII type of undulators $[3,4]$. Apple-II undulators were extensively used to produce variably polarized light at synchrotron radiation sources. The advent of insertion devices that can deliver high brilliance and intense beams of well-controlled variably polarized light has marked a leap type of progress in synchrotron radiation research by widening the range of spectroscopic tools available to users. Strong synergy between theorists and experimental scientist ensures a constant refinement of the quantitative analysis of the data and a widening of the applicability of techniques based on variable polarization. Starting off with pioneering work exploiting the variable degree of linear and circular polarization from angular selected bending magnet radiation $[5,6]$, polarization control has now become an essential tool to study systems with axial anisotropy such as oriented (e.g., chiral or magnetic) or aligned (e.g., antiferromagnetic) systems [7]. Circular or linear dichroism in the spectral distribution (photon energy dependent, measured by absorption or photoemission spectroscopy) or spectral intensity (angular resolved, observed in photoemission spectroscopy) can be measured when exciting core- or valence-electrons in atoms from anisotropic systems by means of radiation with a well defined polarization [7]. Circular and linear polarized light states in fact, present a well-defined orientation or alignment respectively that can probe the anisotropy in matter. Although light can only probe directly the space coordinates of bound electrons, thanks to spin-orbit interaction, circular and linear dichroism are very effective in the analysis of spin states without requiring a spin sensitive detector.

The advent of a VUV-XUV FEL type of source with variable-polarization such as FERMI, is also expected to yield major scientific advances. Experimental activity making use of the, so far uniquely available, native circular polarization has already taken place [8-11]. FEL research in general involves single and multicolor non-linear optics, high coherence for imaging and time resolution. All these features, combined with the availability of circular polarized FEL light are expected to expand the range of circular dichroism experiment that can be made available. Circular dichroism in multi-photon absorption was recently measured in a two-color (pump and probe) photoemission experiment from atomic He [8]. In this type of experiment, orientation in the initially spherical symmetric He atom is induced by a circularly polarized VUV pump photon from the FEL, promoting one electron to an energy level above the He atom ionization potential (IP). This orientation is then probed with virtually zero time delay by further absorption or stimulated emission of one or more circularly polarized infra-red (IR) photon with splitting of the photoemission line into side-bands [12,13], whose intensity and angular distribution depend on the relative orientation of the VUV and IR photon spin [8,14]. This circular dichroism is specific of two-color experiments [14]. The time resolution offered by FERMI opens new perspective in the application of VUV/X-ray circular dichroism to the study of magnetization dynamics. In this path of research dichroism in the coherent diffraction imaging (CDI) of ferromagnetic materials was recently achieved at FERMI [9-11]. Another area of research that can benefit from polarization control of FEL light is biochemistry where molecules with a given geometric orientation (chirality) are studied.

In this work we overview the experimental techniques and the results obtained for the first characterization of the polarization state of a single-pass free electron laser in the VUV. The experiment was performed at FERMI FEL1 operated in the 54-26 nm wavelength range by means of three different techniques. Two experimental techniques were made available at the end-station of beamline DiProI, and one at the end-station of beamline LDM. Each technique was used to characterize the four "pure" polarization states: horizontal, vertical, right-circular and left-circular. 


\section{EXPERIMENTAL METHODS FOR VUV POLARIMETRY AT FERMI}

Benchmark polarization measurements of a new kind of light source such as FERMI need to satisfy several requirements that are difficult to achieve with a single experimental technique. The high brilliance and high longitudinal coherence of the FERMI source, even when compared with other FEL [1] allow a theoretical prediction of the total degree of polarization of the emitted light approaching $100 \%$, for any configuration of the magnetic field of the Apple-II undulators that control the electron trajectory. So far, FERMI has been operated to produce purely linear (horizontal or vertical) or purely circular (right or left) polarization states. Despite the theoretical prediction, the demand for accuracy and completeness of the polarization measurement require an experimental technique that can completely determine the most generic polarization state, which means elliptical, and even capable of detecting residual unpolarized light.

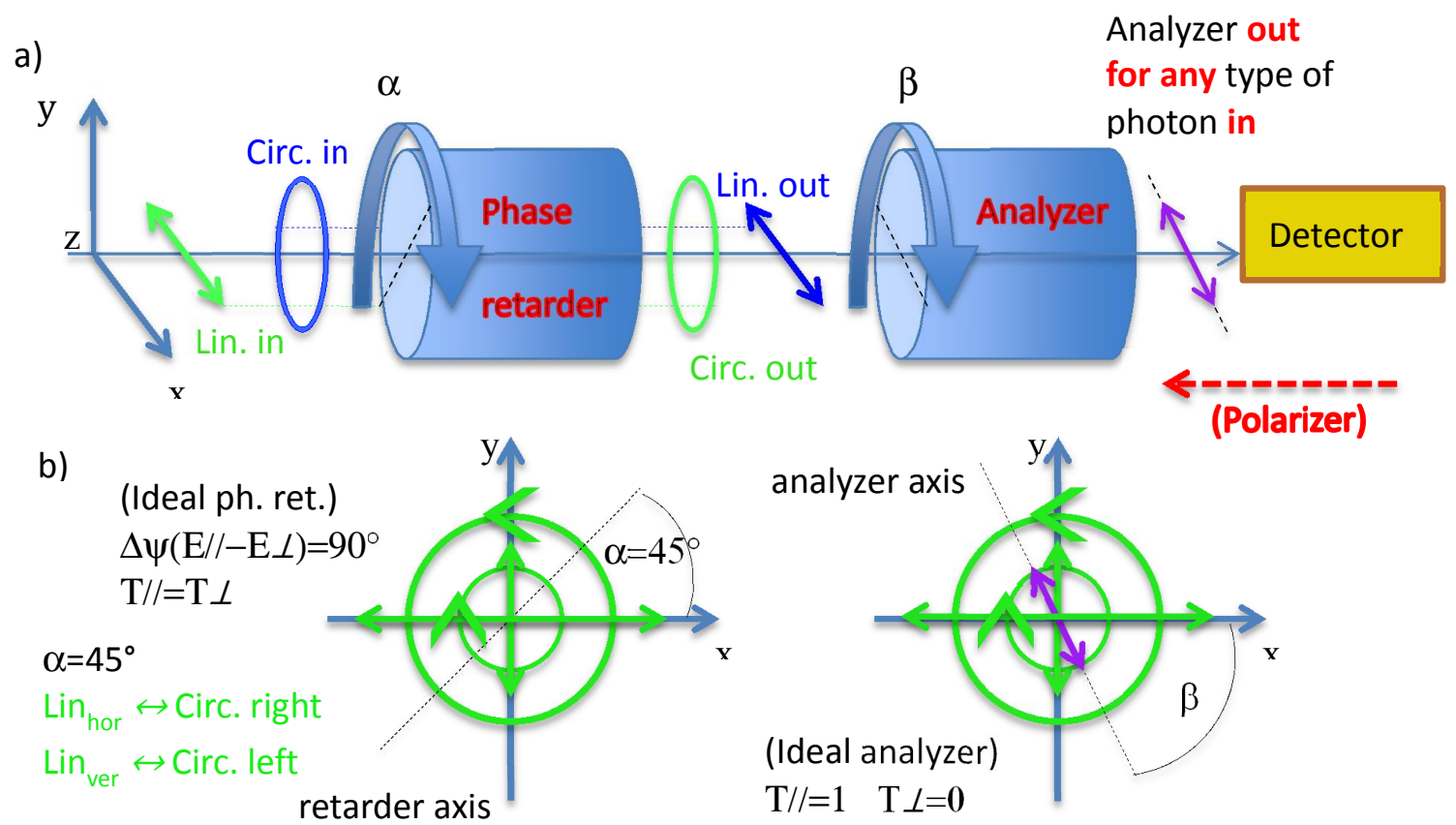

Figure 1. Schematic diagram of an ideal optical polarimeter: a) view parallel to the beam axis; b) view facing the beam axis The beam is transmitted first by a perfect phase retarder; this device is characterized by an isotropic intensity transmission and by a slow (fast) axis so that the electric field component impinging parallel to this axis is retarded (advanced) by exactly a quarter wavelength $\left(90^{\circ}\right)$ relative to the perpendicular one. In this way an incoming circularly polarized photon is transmitted as linearly polarized one directed at $\pm 45^{\circ}$ relative to the phase retarder axis, depending on the helicity of the circular state. Conversely a linearly polarized state aligned at $\pm 45^{\circ}$ relative to the retarder axis is transformed into a circular polarized state with right/left helicity. The retarder axis can be rotated at any angle $\alpha$ (measured in the laboratory reference frame) in the plane of the radiation to match any beam geometry. After phase retardation, the photon travels through a perfect analyzer. This device is characterized by strong anisotropy in the transmission: only the component parallel to the analyzer axis is transmitted. Also this device can be rotated freely (angle $\beta$ ) to sample the whole plane of the radiation. Finally, the transmitted intensity is measured by a suitable detector. The anisotropy in the intensity as a function of $\beta$ depends on the polarization state transmitted by the phase retarder. By reversing the direction of the incident light a polarimeter works as a polarizer.

Among the possible factors that can cause deviation of the actual polarization from ideal values, one must consider machine related issues, such as fluctuations of the electron trajectory, but certainly very important is the impact of transport optics, particularly in the VUV range. At the same time, for the sake of the development of single-shot experiments, or even to 
allow sorting of dichroism data collected by accumulation over multiple shots, polarization measurements at FERMI frequency rate $(10-50 \mathrm{~Hz})$ are a highly desirable feature too.

The problem of polarimetry in the VUV spectral region was initially tackled at synchrotron radiation sources, and the basic principles of the measurements discussed in this work owe in part to this previous expertise [15-19]. Complete determination of the normalized Stokes parameters $\left(s_{1}=\mathrm{S}_{1} / \mathrm{S}_{0}, s_{2}=\mathrm{S}_{2} / \mathrm{S}_{0}, s_{3}=\mathrm{S}_{3} / \mathrm{S}_{0}\right)$, where $\mathrm{S}_{0}$ is the intensity, that describe the polarization state of the light [20], has been performed at several synchrotron radiation facilities by means of optical polarimeters, like the one schematically depicted in Fig. 1 [15-18]. It shares the same basic concept with polatrimeters used in the infrared, visible and near UV range. The key elements are the phase retarder and analyzer that manipulate that polarization state of the incoming radiation in a well-controlled way (see also the caption of Figure 1). High level of accuracy in polarimetry measurements are readily obtained when the phase retarder has the ability of transforming purely linear states into circular ones and vice versa [15-19]. A perfect analyzer transmits only one component of the electrical vector of the incoming radiation. By measuring the total intensity transmitted by the polarimeter for different geometries of the phase retarder and analyzer, one can retrieve the original polarization state of the radiation (unpolarized component included) [15,18]. Perfect phase retarder and analyzers in the shape of transmission filters are easily available for wavelength up to the near UV region. The upper limit of wavelengths that can be probed by transmission filters is determined by the band gap of the polarizing materials. In the UV region, strong absorption prevents the use of transmission filters, multilayers included, and both phase retarder and analyzer rely on the polarizing properties of reflecting surfaces [15-17,19] (see also Figure 2). Optical determination of the polarization state requires a dedicated beamtime allocation, i.e., it is not readily compatible with other experimental activities at a given end-station. On the other hand it is an essential benchmark measurement for a beamline aiming at polarization dependent experiments. Indirect polarization measurements, i.e., from photoemission or photoabsorption data showing strong and well-characterized polarization dependence are also a common practice. In most cases this type of measurement allows to determine separately the degree of linear or circular polarization. Even though such determination is not comprehensive it can be of great practical importance. In any case indirect measurements rely very often on the direct one to provide upper and lower limits for the degree of polarization under scrutiny.

The goal of the first polarization measurements campaign at FERMI is to provide comprehensive and reliable values for the polarization state of light available to users and to assess the sensitivity of the output polarization to machine parameters. For this reason, three dedicated experiments were carried out at two different FERMI beamlines, namely DiProI [9] and LDM. Activity on DiProI is focused on coherent imaging techniques, LDM is the FERMI beamline for atomic, molecular and cluster physics studies [21]. DiProI hosted two experimental setups: an optical VUV polarimeter [15-17,19] (see also Figure 2) and a polarimeter based on the measurement of the angular distribution of photoelectrons (form He $1 s$ core level). This polarimeter makes use of an array of electron time of flight (e- TOF) detectors (see also Figure 5). Both DiProI experiments were carried out at FEL wavelengths of $22 \mathrm{~nm}$ and $36 \mathrm{~nm}$. The experiment carried out on LDM is based on a novel approach in the field of VUV polarimetry, in fact a full Stokes parameters analysis is carried out on long wavelength (visible) light emitted by fluorescence decay from resonantly excited He: a high population of $\mathrm{He}(1 s 3 p)$ atoms is generated by means of FEL light at $53.70 \mathrm{~nm} \quad$ and the polarization analysis is carried out on the fluorescence from the $(\mathrm{He} 1 s 3 p)->\mathrm{He}(1 s 2 s)$ dipole decay channel delivering light at $501.5 \mathrm{~nm}[22,23]$. The experiments carried out at the two beamlines are complementary to each other: experiments on DiProi explore wavelengths that are above the IP of He and compare the results of a VUV polarimeter with the results of a potential shot to shot diagnostic such as the $\mathrm{e}^{-}$TOF photoemission device. The experiment on LDM explores the longer wavelength region below He IP. Also, it provides an assessment of a new VUV polarimetry diagnostic that although limited to the selected wavelengths of appropriate atomic resonances has the major advantage of simplicity and high accuracy. In fact, transmission filters providing perfect phase retardation and analyzing power are readily available for visible light. In the next section we describe the key features of the experimental methods and set-ups. 


\section{EXPERIMENTAL TECHNIQUES AND DATA ANALYSIS METHODS}

\subsection{Wavelength shift fluorescence polarimetry at LDM}

Polarimetry carried out at LDM takes advantage of the polarization properties of fluorescence light from resonantly excited atoms. With this method it is possible to convert polarized UV radiation from the FEL into visible fluorescence light with the same polarization parameters. Figure 2 reports a schematic diagram of the transition involved in the wavelength shifted fluorescence polarimeter that was mounted at the LDM end-station. Full Stokes parameters determination of the visible fluorescence light was carried out by means of a device such as the one schematically described in Figure 1, where phase retarder and analyzer were in the shape of transmission filters.

a)

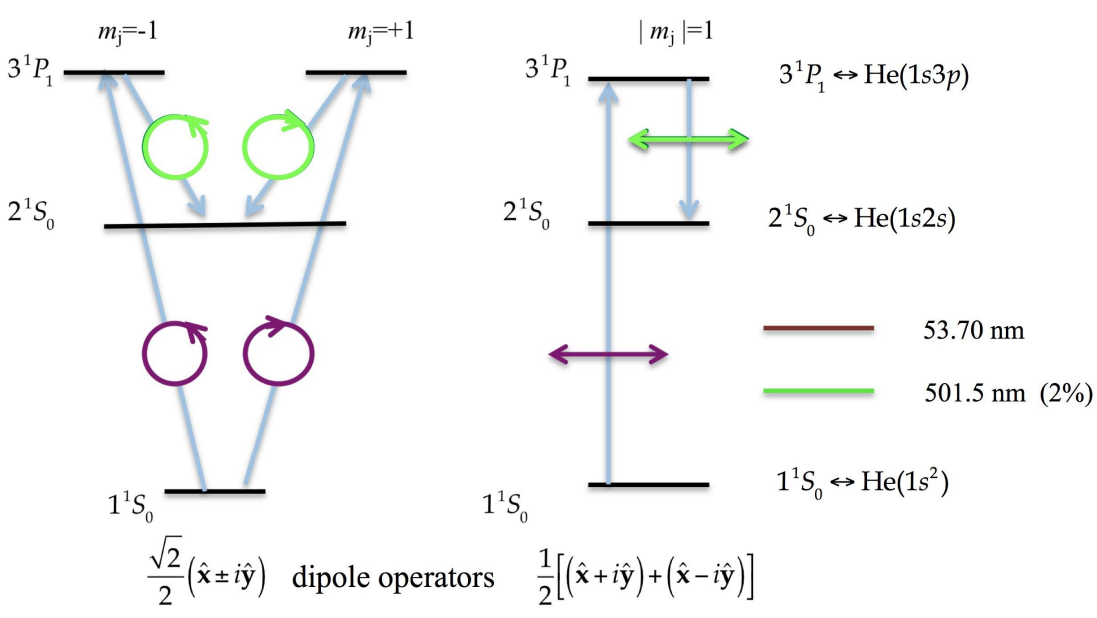

c) Stokes parameters $\left(s_{1}, s_{2}, s_{3}\right)$ from analyzer angular scans (angle $\beta$ ) at given phase retarder angle $(\alpha)$ :

$$
\begin{aligned}
& I\left(\alpha=0^{\circ}+n 90^{\circ}\right)=1+\frac{1}{2} s_{1}[\cos 2 \beta+\cos (4 \alpha-2 \beta)]+s_{3}[\sin 2(\alpha-\beta)] \\
& I\left(\alpha=45^{\circ}+n 90^{\circ}\right)=1+\frac{1}{2} s_{2}[\sin 2 \beta+\cos (4 \alpha-2 \beta)]+s_{3}[\sin 2(\alpha-\beta)]
\end{aligned}
$$

Figure 2. Schematic diagram of the polarization dependent transitions upon resonant excitation of He by means of $53.7 \mathrm{~nm}$ UV light to the $\mathrm{He}(1 s 3 p)$ state $\left(3^{1} P_{1}\right)$, followed by radiative decay to the $\mathrm{He}(1 s 2 s)$ state $\left(2^{1} S_{0}\right)$ with emission of $501.5 \mathrm{~nm}$ fluorescence (visible in the green). Scheme a) excitation by means of circularly polarized light; b) linearly polarized light. The dipole operators and relative $m_{\mathrm{j}}$ selection rules are for quantization of the angular momentum along the beam propagation direction ( $\mathrm{z}$ axis). c) Stokes parameters dependence of the intensity transmitted by a visible light polarimeter, as a function of the rotation $\alpha$ of the phase retarder and $\beta$ of the analyzer (see also Figure 1). The parameter $n$ in the equations is an integer.

Excitation of $\mathrm{He}$ to the $\mathrm{He}(1 s 3 p)$ resonant state by means of polarized light, selectively populates the three-fold $m_{\mathrm{j}}$ degenerate $3^{1} P_{1}$ state. The resonant state can decay by means of two radiative channels: $98 \%$ back to the ground state and $2 \%$ to the state $2^{1} S_{1}$ state corresponding to the $\mathrm{He}(1 s 2 s)$ configuration with emission of green light $(501.5 \mathrm{~nm})$ [22]. The angular momentum quantum numbers of the ground state and those of the intermediate decay channels are identical, thus fluorescence in the green returns the polarization of the primary exciting photon. The polarization of the green fluorescence 
decay light can then be analyzed by means of the aforementioned transmission optics with ideal polarizing properties. Figure 1c) shows the simple formulae that allow to retrieve the Stokes parameters from the angular scans of a visible light polarimeter: when the phase retarder angle $\alpha$ is set to $0+n 90^{\circ}$, where $n$ is an integer, the intensity modulation $I$ measured by scanning the analyzer angle $\beta$ (see also Figure 1) depends only on the Stokes parameters $s_{1}$ and $s_{3}$, while for $\alpha=45^{\circ}+n 90^{\circ}$ the amplitude of the modulation $I$ is determined only by $s_{2}$ and $s_{3}$. An example of wavelength shift fluorescence polarimetry data is shown in Figure 3 (linear horizontal FERMI polarization). Circular (right and left) FERMI polarization states were also measured (data not shown) and the results are summarized in Table 1. In a previous experiment with synchrotron radiation, the wavelength shift method allowed to measure the degree of circular polarization from a bending magnet source. In that case the experiment was performed under Zeeman splitting of the sublevels [22]. The current results prove this feature is inessential for the measurement of $s_{3}[23]$.

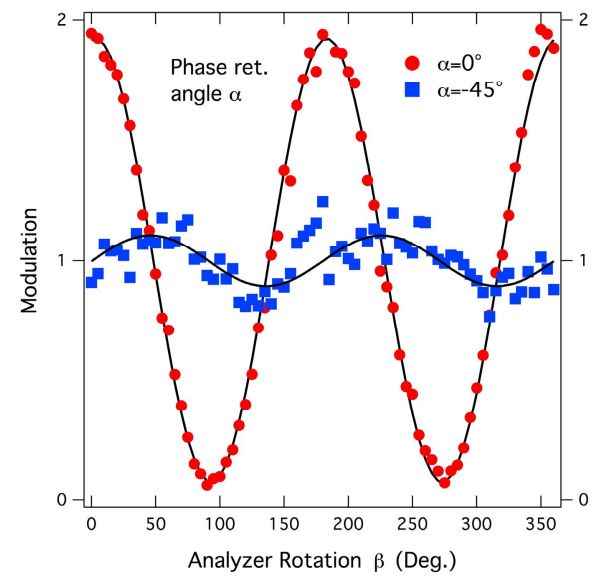

Figure 3. Polarimeter scans by means of analyzer rotation of a FERMI linear horizontal polarization state (nominal $s_{3}=0$ ) at $53.7 \mathrm{~nm}$, measured with fluorescence wavelength shift method at beamline LDM. Red curve: constant phase retarder $\alpha=0^{\circ}$, the modulation is determined by $s_{1}$; blue curve: constant phase retarder $\alpha=45$, the modulation is determined by $s_{2}$. Black solid line: fitting curve by means of the equations given in Figure 2c. The measured values of $s_{1}$ and $s_{2}$ (see table 1) correspond to a tilt of $3^{\circ}$ of the linear state in the laboratory reference frame. The experimental results confirm $s_{3}=0$.

\subsection{The VUV polarimeter}

In a VUV polarimeter phase retardation and polarization analysis are carried out by means of reflection optics. The most critical part is the phase retarder, which needs to satisfy two distinct conditions: phase retardation of the $s$ and $p$ components (defined by the plane of the reflection) approaching $90^{\circ}$ and equal transmission of the two components [15-17,19]. These two conditions are difficult to obtain in the VUV region. The situation is illustrated in Figure 4, which compares the polarizing properties (calculated) of $\mathrm{Au}$ and Mo coated mirrors at $60 \mathrm{eV}$ photon energy (20.6 nm). High phase retardation can be obtained for incidence angles in the region of the Brewster angle, which is near $45^{\circ}$ for the whole VUV range and for any reflecting surface, where extinction of the $p$ component upon reflection also occurs. To overcome this difficulty phase retarders are made to work by phase accumulation from multiple reflection optics [15-17,19]. Improvement in the mirror coating deposition technology allows taking advantage of new and better performing materials for the mirror coating, such as Mo [17,19], while earlier devices relied on Au coating [15,16]. The higher overall reflectivity of Mo compared to $\mathrm{Au}$ in the VUV range allows the use of four mirrors devices [17]. Mo coated mirrors can potentially deteriorate due to oxidation of the coating. This difficulty is overcome by applying thin passive layers. The phase retarder of the VUV polarimeter used at FERMI is in fact a four reflection Mo coated device with capping layer. For a best compromise between $\mathrm{R}_{\mathrm{p}} / \mathrm{R}_{\mathrm{s}}$ approaching 1 and a $90^{\circ}$ degrees phase retardation at the wavelengths under study, the angle of incidence was set to $20^{\circ}$. Prior to installation at beamline DiProI, this phase retarder was characterized at the synchrotron source BESSYII. The analyzer is made of a single uncoated fused silica mirror at $45^{\circ}$. The full data analysis method for VUV polarimetry measurements was previously reported in the literature [16-18] and in this paper a simple qualitative discussion is given. Like visible light polarization analysis, the dependence of the transmitted intensity $I$ can be derived from Müller matrices formalism [16-18], describing the modification of the polarization state by transmission through phase retarder and 
analyzer. Unlike the case of visible light, with a VUV polarimeter the transmission curve $I$ depends on all the Stokes parameters for any value of the angle rotation angle $\alpha$ and $\beta$ and on the polarizing properties $\mathbf{P}$ of the phase retarder i.e., $I=I\left(\mathbf{P}, \alpha, \beta, s_{1}, s_{2}, S_{3}\right)[16-18]$. In other words a larger number of fitting terms and a larger dataset is required to retrieve the Stokes parameters, nevertheless, reflection VUV polarimetry was successfully used at several synchrotron radiation facilities [15-17].

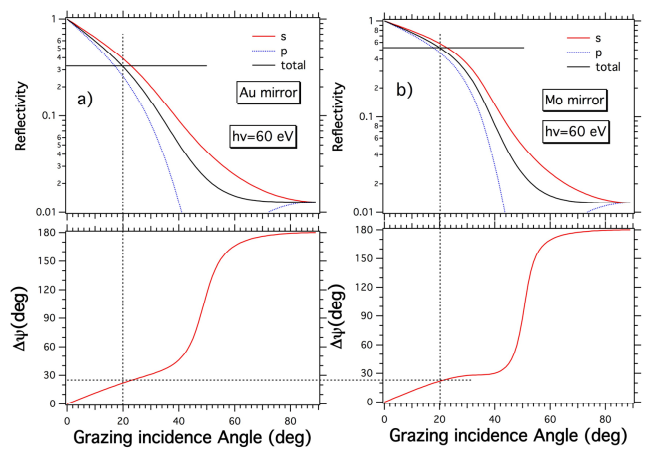

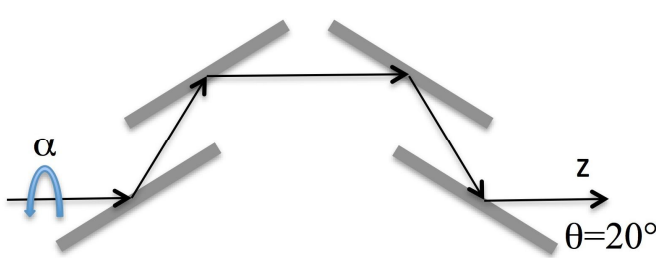

c)

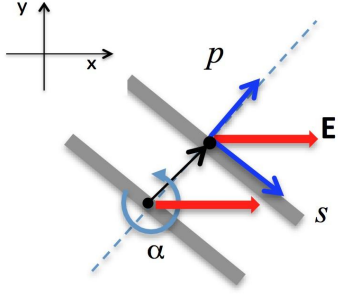

d)

Figure 4. Reflectivity and phase retardation of a single mirror coated with a) Au and b) Mo. The optical constants for this calculation are from [24]. Schematic diagram of a four-reflection phase retarder: c) view parallel to the beam, d) section of the device in a plane perpendicular to the beam. With this device the angle of incidence $\theta$ of the light is the same at each reflection and the incoming and outgoing beams are coaxial (panel c). The whole device can rotate around the beam axis by any angle $\alpha$. In in this way one can vary the $s$ (perpendicular to the plane of the reflection) and $p$ (parallel to the plane of the reflection) decomposition of the electrical vector $\mathbf{E}$ (panel d).

\subsection{The $\mathrm{e}^{-}$TOF polarimeter}

This technique provides a direct measurement of the degree of linear polarization from photoemission experiments. In fact, the linear component produces strong anisotropy in the photoelectron angular distribution (see Figure 5). Unlike the fluorescence and VUV polarimeter the e- TOF cannot distinguish circularly polarized light from unpolarized light, as these states give the same (isotropic) contribution to photoelectron angular distribution [25]. However with a source such as FERMI where the total degree of polarization is equal (or extremely close to) $100 \%$, the assumption that isotropic emission is due only to the circular components is reasonable and the degree of circular polarization can be estimated (see Figure $5 b)$. The exact shape of the anisotropy curve depends on the photoemission dynamics parameter $\beta$, which, in turn is specific of the core level under study. In this experiment photoemission from ground state He was used. One photon photoemission from $\mathrm{He} 1 s$ is characterized by an asymmetry parameter $\beta=2$, that is the value that ensures the largest amount of anisotropy that is physically available [25]. The $\mathrm{e}^{-}$TOF type of spectrometer is a relatively simple and compact (highly directional) electron kinetic energy analyzer that can be used with short-pulsed light sources where the time 0 of the photoemission process can be determined very accurately. For the polarization measurements at FERMI a circular arrangement of sixteen and coplanar $\mathrm{e}^{-} \mathrm{TOFs}$, mounted on a plane perpendicular to the beam propagation direction was used [26]. With this geometry, FERMI single shot photoelectron angular distributions can be measured, yielding polarization parameters with 
exactly the FERMI repetition rate. The photoelectron based-technique appears to be particularly suited to monitor beam polarization as a function of fast changing machine parameters, or to monitor fluctuations that may be correlated to FEL electron beam position monitor data.

a)

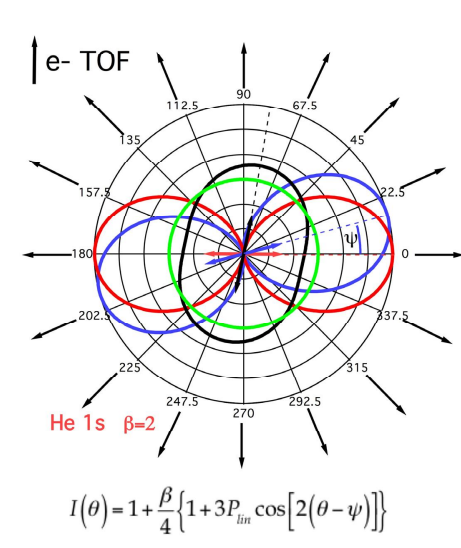

b)

$$
P_{l i t}=\frac{I(\psi)-I(\psi \pm \pi / 2)}{I(\psi)+I(\psi \pm \pi / 2)}
$$

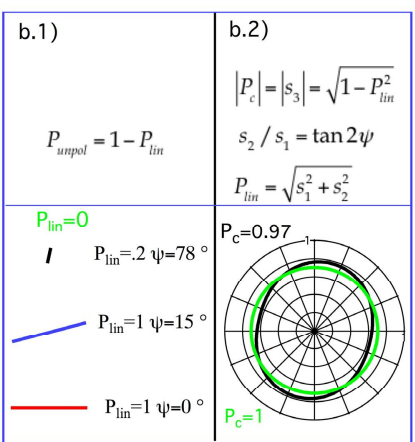

Figure 5. a) Schematic diagram of a the $\mathrm{e}^{-}$TOF polarimeter. A circular arrangement of sixteen coplanar $\mathrm{e}^{-}$TOFs sample the photoemission angular distribution $I(\theta)(\theta$ is in the laboratory reference frame) in a plane perpendicular to the beam axis [24]. Anistropy in the angular distribution is directly related to the degree of linear polarization via the photoemission parameter $\beta$, which is specific of the given core level under study (for $\mathrm{He} 1 s \beta=2$ ) [25]. Unpolarized or circularly polarized light provide the same type of isotropic emission. However, with a high polarization source such as FERMI one can assume that isotropic emission is due uniquely to circular polarized light. With this assumption one can retrieve the full polarization state parameters (see panel b.2). The polarization states shown in this figure are examples.

\section{SUMMARY OF EXPERIMENTAL RESULTS}

\begin{tabular}{|c|c|c|c|c|c|}
\hline Wavelength (nm) & Nominal FEL Pol. & Tot. Pol. (measured) & $s_{1}$ & $s_{2}$ & $s_{3}$ \\
\hline 53.70 & Linear Hor. & 0.92 & 0.92 & 0.11 & 0.07 \\
\hline 53.70 & Circ. right & 0.92 & -0.07 & 0.21 & 0.89 \\
\hline 53.70 & Circ. Left & 0.93 & -0.20 & -0.31 & -0.85 \\
\hline
\end{tabular}

Table 1. Results from the fluorescence polarimeter

\begin{tabular}{|c|c|c|c|c|c|}
\hline Wavelength (nm) & Nominal FEL Pol. & Tot. Pol. (measured) & $s_{1}$ & $s_{2}$ & $s_{3}$ \\
\hline 26 & Linear Vert. & 0.97 & -0.97 & 0.03 & 0.07 \\
\hline 26 & Circ. right & 0.96 & -0.02 & 0.05 & 0.96 \\
\hline 32 & Lin. Vert. & 0.96 & -0.96 & 0.02 & 0.06 \\
\hline 32 & Circ. Left & 0.93 & -0.05 & -0.19 & -0.91 \\
\hline
\end{tabular}

Table 2. Results from the VUV polarimeter

\begin{tabular}{|c|c|c|c|c|c|c|}
\hline Wavelength $(\mathrm{nm})$ & Nominal FEL Pol. & $\mathrm{P}_{\text {lin }}$ & tilt $\Psi($ deg.) & $s_{1}$ & $s_{2}$ & $S_{3}$ \\
\hline 26 & Linear Vert. & 0.97 & $89.7 \pm 1$ & -0.97 & 0.01 & 0.25 \\
\hline 26 & Linear Hor. & 0.94 & $0.4 \pm 1$ & 0.94 & 0.01 & 0.34 \\
\hline 26 & Circ. Left & 0.11 & $50.0 \pm 6$ & -0.02 & 0.11 & -0.99 \\
\hline 26 & Circ. Right & 0.11 & $127.0 \pm 6$ & -0.03 & -0.10 & 0.99 \\
\hline 32 & Linear Vert. & 0.90 & $91.3 \pm 1$ & -0.90 & -0.04 & 0.43 \\
\hline 32 & Linear Hor. & 0.97 & $-1.2 \pm 1$ & 0.97 & -0.04 & 0.23 \\
\hline 32 & Circ. Left & 0.10 & $124.0 \pm 5$ & -0.04 & -0.09 & -0.99 \\
\hline 32 & Circ. Right & 0.14 & $53.0 \pm 6$ & -0.04 & 0.13 & 0.99 \\
\hline
\end{tabular}

Table 3. Results from the $\mathbf{e}^{-}$TOF polarimeter 
The results of the polarization measurement at FERMI are summarized in Tables 1-3. The Stokes parameters reported in these tables are all normalized to the intensity (Stokes parameter $\mathrm{S}_{0}$ ), thus

$$
\sqrt{{s_{1}}^{2}+{s_{2}}^{2}+{s_{3}}^{2}}=P_{\text {tot }}
$$

where, $P_{\text {tot }}$ is the total degree of polarization. Note that $\mathrm{e}^{-}$TOF polarimeter measures directly the degree of linear polarization $P_{l i n}$ and the tilt of the linear component $\Psi$, which corresponds to a direct measurement of the Stokes parameters $s_{1}$ and $s_{2}$. The degree of circular polarization $s_{3}$ reported in Table 3 is calculated by means of $(1)$, under the assumption that $P_{t o t}=1$ (see also the formulae in Figure 5b). The first remark is that all measurements carried out with the three different polarimeters on linear states are consistent with the predicted high degree of total polarization of the FERMI FEL source. The results from setups that measure directly the degree of circular polarization, namely the fluorescence and the VUV polarimeter, show that FERMI can perform high quality circular dichroism experiments both in terms of polarization and polarization reversal. The results from all techniques are consistent with a non-negligible residual of linear polarization from nominally circular states, which is measured by the parameter $s_{1}$ and $s_{2}$. Thus the nominal circular state is actually elliptical [20]. The effect is more pronounced at long wavelength (see Table 1). The linear state observed along with the nominally pure circular one has a defined trend: the value of $s_{1}$ is always negative the value of $s_{2}$ switches from positive to negative along with $s_{3}$, which means along with the circular polarization. These are the Stokes parameters of elliptical states with the major axis closer to the vertical direction and with a mirroring switching along the same vertical direction [20] (see also Figure 7).

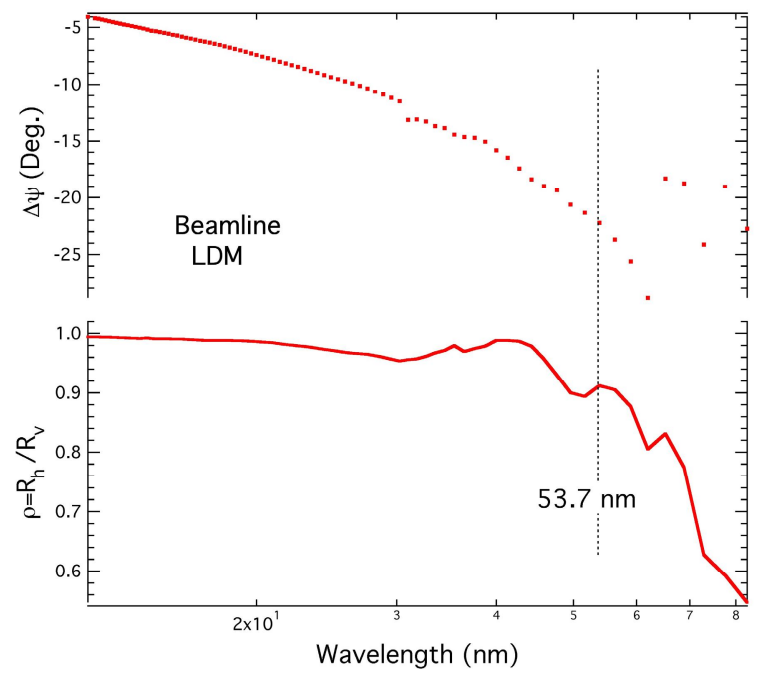

Figure 6. Phse retardation $\Delta \psi$ and reflectivity ratio $\rho$ between the horizontal and vertical component of the electrical field upon transmission through beamline LDM, as derived from Fresnel equations. On LDM a total of of seven reflections steer and focus the beam from the source to the end station. $\mathrm{C}$ coated mirrors: four horizontally steering with incidence (glancing ) angles: $5^{\circ}(\mathrm{x} 1), 2.5^{\circ}(\mathrm{x} 2), 2^{\circ}(\mathrm{x} 1)$; Au coated mirrors: two veertically steering (one focusing) with incidence angle $2^{\circ}$ and one horizontally steering (and focusing) at $2^{\circ}$ glancing incidence. Beamline DiProI is composed by six optical elements: three C coated deflecting horizontally with incidence angles angles $5^{\circ}(\mathrm{x} 1), 2.5^{\circ}(\mathrm{x} 2)$ and three Au coated sharing the same parameters with the LDM ones. The database of optical constants used for these calculations are as follows: Au from ref. [24] (whole wavelength range); C from [24] (wavelength range $31 \mathrm{~nm}-80 \mathrm{~nm}$ ) and from [27] (wavelength range $12 \mathrm{~nm}-31 \mathrm{~nm}$ ).

This is the typical trend when circular states (with opposite helicity) are transmitted by birefingent optics set to a given geometry. The birefringent system in this case can be identified with the photon beam transport system. Figure 6 shows the theoretical polarizing properties of beamline LDM (see also caption for more details on the calculations). The beamline polarization properties descend both from the asymmetry in the reflectivity of the horizontal and vertical component 
( $\rho=R_{h} / R_{v}$ ) and from phase retardation of the components $\Delta \psi$. The asymmetric reflectivity (parameter $\rho$ ) transforms an initially circular state into an elliptical one with vertical major axis, the phase retardation $\Delta \psi$ tilts the axis of the ellipse. Figure 7 compares the elliptical states measured at beamline LDM by means of fluorescence polarimetry and the theoretical ellipses due to transmission of $53.7 \mathrm{~nm}$ circular states through a polarizing device with parameters obtained from the calculation.
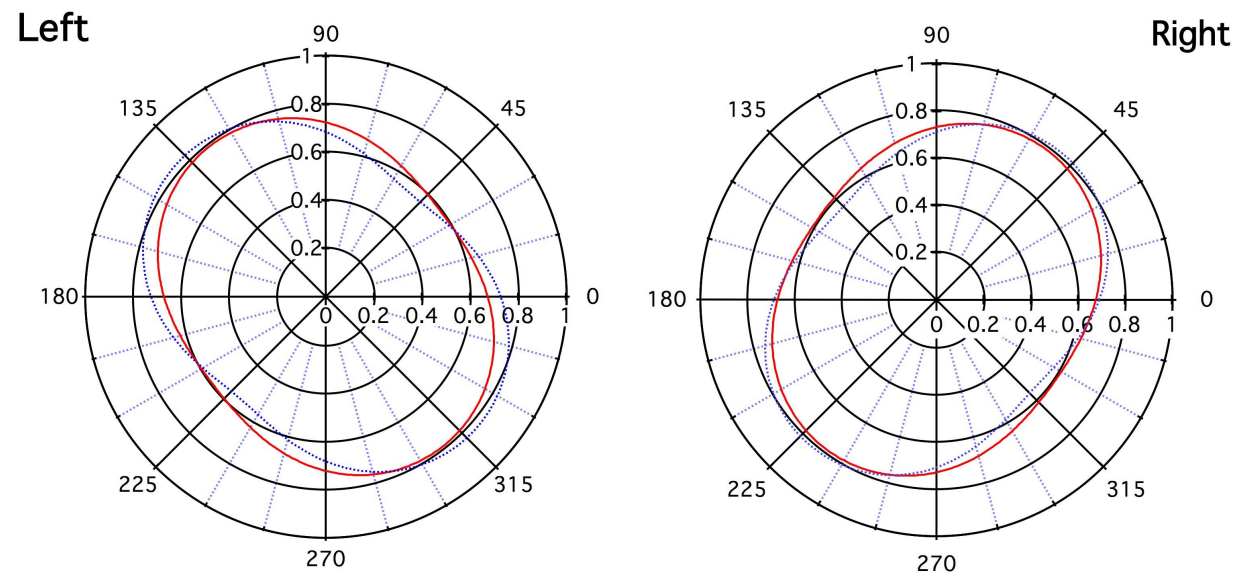

Figure 7. Comparison between elliptical states measured at $53.7 \mathrm{~nm}$ at the LDM end-station (red solid line-Stokes parameters in table 1) and elliptical states originated by transmission of an initially circular state $\left(s_{3}= \pm 1\right)$ according to the theoretical LDM polarizing properties shown in figure 6 (blue dotted line). By applying Müller matrix formalism [15-19], the resulting theoretical Stokes parameters are: $s_{1}=-0.09, s_{2} \pm 0.37, s_{3}= \pm 0.92$.

\section{CONCLUSIONS}

The Stokes parameters of VUV light produced by FERMI FEL1 and available at experimental stations LDM and DiProI have been thoroughly characterized for different settings of the polarization at the source. The approach to the experiment was to make use of multiple techniques. The rationale behind this approach is to provide reliable measurements in different regions of the UV range and at the same time to test the feasibility of non-optical techniques with the potential for a singlepulse polarization analysis. Polarimetry in the UV region of the spectrum is in fact non trivial and setups spanning a long wavelength region may be obtained at the cost of accuracy and sensitivity. Single pulse polarimetry from a variable polarization FEL source was tested for the first time. The independent results are consistent with one another, and they all point out at the very high degree of polarized light available at the experiment. Stokes parameters analysis and theoretical calculations show that circular states at the source are transmitted to the experiment as elliptical due to the polarizing properties of the photon beam transport optics. The effect is more pronounced at long wavelength but even in this case a high degree of circular polarization for dichroism experiments was measured.

\section{ACKNOWLEDGEMENTS}

We acknowledge the contribution of the entire FERMI team that made possible these measurements with continuous support in running the free electron laser. We wish to thank M. Lonza, R. Borghes and L. Pivetta for their support in the integration of the instruments with the FERMI control system, and M. Kiskinova, Z. Huang and A. Lutman who contributed with many insightful discussions. The authors are indebted to M. Svandrlik and F. Parmigiani for their advice and suggestions. MC and GDN acknowledge support by the Italian-Slovenian Crossborder Cooperation Programme (CITIUS project, 2013/2010090600115298). MI acknowledges financial support from the Volkswagen foundation within the Peter Paul Ewald-Fellowship. AKn thanks A. Ehresmann for the support received and acknowledge the contribution of the State Initiative for the Development of Scientific and Economic Excellence (LOEWE) in the LOEWE-Focus ELCH. 
GL, BV, JL, PZ acknowledge financial support from the Triangle de la physique, under contracts IMMAGE and POLIMMAGE, for the realization of the mirror based polarizer. GL and PZ acknowledge the ESF COST Action MP1203 for partial financial support. The resources that made possible the construction of FERMI were obtained and managed by G. Comelli, A. Franciosi and C. Rizzuto. This work was funded by the FERMI project of Elettra-Sincrotrone Trieste, partially supported by the Ministry of University and Research (grant nos FIRB-RBAP045JF2 and FIRB-RBAP06AWK3).

\section{REFERENCES}

[1] E. Allaria, et al., "Highly coherent and stable pulses from the FERMI seeded free-electron laser in the extreme ultraviolet," Nature Photon. 6, 699-704 (2012).

[2] E. Allaria, D. Castronovo, P. Cinquegrana, P. Craievich, M. Dal Forno, M. B. Danailov, G. D'Auria, A. Demidovich, G. De Ninno, S. Di Mitri, et al., "Two-stage seeded soft-X-ray free-electron laser," Nature Photon. 7, 913-918 (2013).

[3] S. Sasaki, "Analyses for a planar variably-polarizing undulator," Nucl. Instrum. Meth. Phys. Res. A 347, 83-86 (1994).

[4] A. B. Temnykh, "Delta undulator for Cornell energy recovery linac," Phys. Rev. ST Accel. Beams 11, 120702 (2008).

[5] G. Schütz, W. Wagner, W. Wilhelm, P. Kienle, "Absorption of Circularly Polarized X Rays in Iron," Phys. Rev. Lett. 58, 737-740 (1987).

[6] C.T. Chen, F. Sette, Y. Ma, S. Modesti, "Soft-x-ray magnetic circular dichroism at the L2,3 edges of nickel," Phys Rev. B 42, 7262 (1990).

[7] J.B. Kortright, D.D. Awschalom, J. Stöhr, S.D. Bader, Y.U. Idzerda, S.S.P. Parkin, I.K. Schuller, H.-C. Siegmann, "Research frontiers in magnetic materials at soft X-ray synchrotron radiation facilities," Journal of Mag. and Mag. Mat. 207, 7-44 (1999).

[8] T. Mazza, M. Ilchen, A. J. Rafipoor, C. Callegari, P. Finetti, O. Plekan, K. C. Prince, R. Richter, M. B. Danailov, A. Demidovich, et al., "Determining the polarization state of an extreme ultraviolet free-electron laser beam using atomic circular dichroism," Nature Commun. 5, 3648 (2014).

[9] F. Capotondi, E. Pedersoli, N. Mahne, R. H. Menk, G. Passos, L. Raimondi, C. Svetina, G. Sandrin, M. Zangrando, M. Kiskinova, et al., " Coherent imaging using seeded free-electron laser pulses with variable polarization: First results and research opportunities," Rev. Sci. Instrum. 84, 051301 (2013).

[10] L. Müller, S. Schleitzer, C. Gutt, B. Pfau, S. Schaffert, J. Geilhufe, C. von Korff Schmising, M. Schneider, C. M. Günther, F. Büttner, et al., "Ultrafast Dynamics of Magnetic Domain Structures Probed by Coherent Free-Electron Laser Light," Synchrotron Radiation News 26, 27-32 (2013).

[11]] C. von Korff Schmising, B. Pfau, M. Schneider, C. M. Günther, M. Giovannella, J. Perron, B. Vodungbo, L. Müller, F. Capotondi, E. Pedersoli, N. Mahne,J. Lüning, and S. Eisebitt, "Imaging ultrafast demagnetization dynamics after a spatially localized optical excitation," Phys. Rev. Lett. 112, 217203 (2014).

[12] T. E. Glover, R. W. Schoenlein, A. H. Chin, C. V. Shank, "Observation of laser assisted photoelectric effect and femtosecond High Order Harmonic Radiation," Phys. Rev. Lett. 76, 2468-2471 (1996).

[13] M. Meyer, J. T. Costello, S. Düsterer, W. B. Li, P. J. Radcliffe, "Two-colour experiments in the gas phase," J. Phys. B 43, 194006 (2010).

[14] A. K. Kazansky, A. V. Grigorieva, N. M. Kabachnik, "Dichroism in short-pulse two-color XUV plus IR multiphoton ionization of atoms," Phys. Rev. A 85, 053409 (2012).

[15] A. Gaupp, M. Mast,"First experimental experience with a VUV polarimeter at BESSY," Rev. Sci. Instrum. 60, 2213-2215 (1989).

[16] T. Koide, T. Shidara, M. Yuri, N. Kandaka, K. Yamaguchi, H. Fukutani, "Elliptical-polarization analyses of synchrotron radiation in the 5-80-eV region with a reflection polarimeter," Nucl. Instrum. Methods A 308, 635644 (1991).

[17] H. Höchst, R. Patel, F. Middleton, "Multiple-reflection $\lambda / 4$ phase shifter: a viable alternative to generate circular-polarized synchrotron radiation," Nucl. Instrum. Methods Phys. Res. A 347, 107-114 (1994).

[18]F. Schäfers, H.-C. Mertins, A. Gaupp, W. Gudat, M. Mertin, I. Packe, F. Schmolla, S. Di Fonzo, G. Soulli, W. Jark, R. Walker, X. Le Cann, R. Nyholm, M. Eriksson, ,"Soft X-ray polarimeter with multilayer optics: complete analysis of the polarization state of the light," Appl. Opt. 38, 4074-4088 (1999). 
[19]B. Vodungbo, A. Barszczak Sardinha, J. Gautier, G. Lambert, C. Valentin, M. Lozano, G. Iaquaniello, F. Delmotte, S. Sebban, J. Lüuning, and P. Zeitoun, , "Polarization control of high order harmonics in the EUV photon energy range," Opt. Expr. 19, 4346-4356 (2011).

[20] M. Born and E. Wolf, [Principles of Optics Sixth Edition], Pergamon Press, Oxford, 23-32 (1989).

[21] V. Lyamayev, Y. Ovcharenko, R. Katzy, M. Devetta et al., "A modular end-station for atomic, molecular, and cluster science at the Low Density Matter beamline of FERMI@Elettra," J. Phys. B: At. Mol. Opt. Phys. 46, 164007-1640015 (2013).

[22] C. Latimer, M. MacDonald, and P. Finetti, "A new method for polarization analysis in the VUV," J. Electron Spectrosc. Relat. Phenom. 101-103, 875 (1999).

[23] V. Y. Backman, S. V. Bobashev, and O. S. Vasyutinskii, "Determination of polarization of vacuum-ultraviolet radiation by fluorescence and by probe-beam tchnique," Sol. Phys. 164, 397-401 (1996).

[24] B. Henke, E. Gullikson, and J. Davis, "X-ray interactions: photoabsorption, scattering, transmission, and reflection at E=50-30000 eV, Z=1-92," At. Data Nucl. Data Tables 54, 181-342 (1993).

[25] S. T. Manson and A. F. Starace, "Photoelectron angular distributions: energy dependence for s- subshells," Rev. Mod. Phys. 54, 389-405 (1982).

[26] J. Viefhaus et al., In preparation.

[27]E. D. Palik, ed., [Handbook of Optical Constants of Solids, Vol. 1, Vol. 2 and Vol. 3], Academic Press, (1997). 